\title{
Reciprocal regulation of iNOS and PARP-1 during allergen-induced eosinophilia
}

\author{
A.S. Naura*, R. Datta*, C.P. Hans*, M. Zerfaoui*, B.M. Rezk*, Y. Errami*, \\ M. Oumouna*, K. Matrougui ${ }^{\#}$ and A.H. Boulares*
}

ABSTRACT: Inducible nitric oxide synthase (iNOS) inhibition was recently shown to exert no effect on allergen challenge in human asthma, raising serious concerns about the role of the protein in the disease. The present study investigated the role of iNOS in ovalbumin-induced eosinophilia from the perspective of its relationship with poly(ADP-ribose) polymerase-1 (PARP-1) and oxidative DNA damage.

A mouse model of ovalbumin-induced eosinophilia was used to conduct the studies.

iNOS-associated protein nitration and tissue damage were partially responsible for allergeninduced eosinophilia. iNOS expression was required for oxidative DNA damage and PARP-1 activation upon allergen challenge. PARP-1 was required for iNOS expression and protein nitration, and this requirement was connected to nuclear factor-кB. PARP-1 was an important substrate for iNOS-associated by-products after ovalbumin-challenge. PARP-1 nitration blocked its poly(ADP-

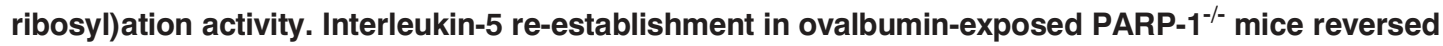
eosinophilia and partial mucus production without a reversal of iNOS expression, concomitant protein nitration or associated DNA damage.

The present results demonstrate a reciprocal relationship between inducible nitric oxide synthase and poly(ADP-ribose) polymerase-1 and suggest that expression of inducible nitric oxide synthase may be dispensable for eosinophilia after interleukin-5 production. Inducible nitric oxide synthase may be required for oxidative DNA damage and full manifestation of mucus production. Such dispensability may explain, in part, the reported ineffectiveness of inducible nitric oxide synthase inhibition in preventing allergen-induced inflammation in humans.

KEYWORDS: Allergy, cytokines, eosinophils, inflammation, lung, transgenic/knockout mice

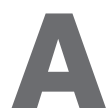
sthma is a complex chronic inflammatory disease of the airways whose prevalence and morbidity is increasing worldwide at an alarming rate [1]. The amount of nitric oxide (NO) in exhaled air is increased in individuals with asthma and this amount reflects disease severity $[2,3]$. NO is thought to play conflicting roles in airway physiology and pathophysiology $[4,5]$. Indeed, NO plays a major beneficial role in airway function as it controls vascular and bronchial tone and neuroendocrine regulation of airway mediator release [3]. However, after combining with superoxide to form the highly reactive peroxynitrite $\left(\mathrm{ONOO}^{-}\right)$, $\mathrm{NO}$ rapidly oxidises sulphydryl groups and mediates nitration and hydroxylation of aromatic compounds including tyrosine, tryptophan, and guanosine [5]. The latter molecular changes may participate in cell demise and ultimate tissue injury. NO is synthesised by a variety of cell types, including (activated) macrophages as well as endothelial and epithelial cells. The synthesis of $\mathrm{NO}$ is catalysed by each of three distinct forms of $\mathrm{NO}$ synthase (NOS): neuronal NOS (nNOS); endothelial NOS (eNOS); and inducible NOS (iNOS). Whereas iNOS is either absent or present in only small amounts in most cell types and tissues under normal circumstances, a wide range of inflammatory agents, including allergen exposure, rapidly induces its synthesis $[4,6]$.

Although inhibition of NO production by iNOS appeared to be a very viable therapeutic target to prevent manifestation of asthma symptoms upon exposure to allergens [7-9], a recent clinical study by SINGH et al. [10] raised serious questions about the validity of such a strategy. SingH et al. [10] reported that iNOS inhibition with GW274150, a selective and potent iNOS inhibitor, effectively reduces exhaled breath $\mathrm{NO}$ but does not affect airway inflammatory cell numbers or airway hyperreactivity after allergen challenge in subjects with asthma. Interestingly, reports by Holla et al. [11] and BATRA et al. [12] show that polymorphisms in the iNOS gene may be important for asthma protection or susceptibility. Such conflicting
AFFILIATIONS

*Dept of Pharmacology and

Experimental Therapeutics, Louisiana State University Health Sciences Center, and

\#Dept of Physiology, Tulane University Medical Center, New Orleans, LA, USA.

CORRESPONDENCE

A.H. Boulares

Louisiana State University Health

Sciences Center

Dept of Pharmacology and

Experimental Therapeutics

1901 Perdido St.

New Orleans

LA 70112

USA

Fax: 15045682361

E-mail: hboulr@Isuhsc.edu

Received:

June 122008

Accepted after revision:

September 152008

SUPPORT STATEMENT

Supported, in part, by grants HL072889 and 1P20RR18766

(overall project director D. Kapusta) from the National Institute of Health (Bethesda, MD, USA) and by funds from the Louisiana Cancer Research Consortium (New Orleans, LA, USA) to $\mathrm{H}$. Boulares.

STATEMENT OF INTEREST

None declared.

European Respiratory Journal

Print ISSN 0903-1936

Online ISSN 1399-3003 
reports undoubtedly suggest that additional studies are necessary to fully establish the intricate role(s) of $\mathrm{NO}$ and its metabolites during airway inflammation. Furthermore, it is becoming clear that an examination of the role of iNOS in the context of other players in airway inflammation is necessary. This approach may not only allow for the understanding of the role of iNOS during inflammation, but may also identify alternative strategies for the treatment of asthma symptoms.

The present authors' group and others have shown the involvement of poly(ADP-ribose) polymerase (PARP)-1 in tissue injury and its implication in several conditions associated with oxidative stress and inflammation including allergic airway inflammation [13-16] (reviewed in [17]). In addition to its effects on cell and tissue homeostasis through nicotinamide adenine dinucleotide $\left(\mathrm{NAD}^{+}\right)$metabolism, PARP-1 is thought to participate in inflammation by regulating, directly or indirectly, the expression of several inflammatory factors including iNOS (reviewed [17-19]). Such activity has been associated with the ability of PARP-1 to regulate signal transduction events that result in the activation of nuclear factor (NF)- $\kappa \mathrm{B}[20,21]$.

The current study examined the role of iNOS in allergeninduced eosinophilia from the perspective of its relationship with PARP-1 and oxidative DNA damage, revealing a reciprocal relationship between the two proteins. The present results show that PARP-1 is required for iNOS expression and is activated by oxidative DNA damage caused by iNOS byproducts, and that PARP-1 enzymatic activity is modulated by nitration. Furthermore, the current data show that iNOS may be dispensable after interleukin (IL)-5 production. Such dispensability may explain the reported ineffectiveness of a specific iNOS inhibitor in preventing allergen-induced inflammation in humans [10].

\section{METHODS}

\section{Animals}

Mice were bred in a specific-pathogen free facility at Louisiana State University Health Sciences Center (LSUHSC; New Orleans, LA USA), and allowed unlimited access to sterilised chow and water. Maintenance, experimental protocols, and procedures were all approved by the LSUHSC Animal Care \& Use Committee. C57BL/6 wild-type (WT) and iNOS $^{-/-}$mice were purchased from Jackson Laboratories (Bar Harbor, ME, USA). C57BL/6 PARP-1 $^{-/-}$mice were generated by backcrossing the knockout mice under C57BL/6xSV129 mixed background with C57BL/6 WT mice for at least seven generations. The last generation was interbred to generate the C57BL/ 6 PARP-1 ${ }^{-/-}$mice.

\section{Protocols for sensitisation, challenge, organ recovery and tissue staining}

Age 6-8 weeks C57BL/6 WT, $\mathrm{iNOS}^{-/-}$or PARP-1/- mice were sensitised and challenged as previously described [22].

Animals were killed by $\mathrm{CO}_{2}$ asphyxiation and lungs were fixed with formalin for histological analysis or subjected to bronchioalveolar lavage. Formalin-fixed lungs were sectioned and subjected to haematoxylin and eosin, periodic acid-Schiff staining using standard protocols or to immunohistochemistry (IHC) with the appropriate antibodies as described previously [13].

\section{Cell culture, immunofluorescence microscopy, immunoblot analysis and immunoprecipitation}

Naïve peritoneal macrophages were isolated using a standard protocol and cultured in RPMI medium supplemented with antibiotics and $10 \%$ foetal bovine serum. Cells were then seeded on chamber slides and treated with $1 \mu \mathrm{g} \cdot \mathrm{mL}^{-1}$ lipopolysaccharide (LPS; Alexis Biochemicals, San Diego, CA, USA) for different time intervals. Cells were then fixed, permeabilised and stained with antibodies to murine p65 NF- $\kappa \mathrm{B}$ and with 4'-6-diamidino-2-phenylindole essentially as described [23]. They were then examined with a Nikon fluorescence microscope. Immunoblot analysis was conducted essentially as described [23]. Nitrocellulose filters were then probed with antibodies to iNOS, PARP-1 or actin (Santa Cruz Biotechnology, Santa Cruz, CA, USA). Immune complexes were detected with appropriate secondary antibodies and chemiluminescence reagents (Pierce, Rockford, IL, USA). Immunoprecipitation with antibodies to nitrotyrosine was conducted essentially as described [24].

\section{Data analysis}

All data are expressed as mean \pm SD of values from $\geqslant 6$ mice per group, unless otherwise stated. Differences between experimental groups were analysed by one way ANOVA followed by Dunnett's multiple comparison test.

\section{RESULTS}

iNOS-associated protein nitration and tissue damage are partially responsible for allergen-induced eosinophilia following exposure

Although the role of iNOS in the pathogenesis of allergeninduced airway inflammation has yet to be fully elucidated [25], it is widely accepted that the potential mechanism by which iNOS contributes to disease pathogenesis is through the mediation of tissue damage as a result of the excessive production of $\mathrm{NO}$ and its by-product $\mathrm{ONOO}^{-}$. Accordingly, the present study began by addressing the association between iNOS-mediated tissue damage and airway inflammation. It was found that ovalbumin (OVA) sensitisation and challenge were followed by airway inflammation manifested as eosinophilia (fig. 1a-f). Such an inflammatory response coincided with robust iNOS expression and concomitant protein nitration-associated tissue damage as assessed by IHC with antibodies to iNOS or nitrotyrosine (fig. $1 \mathrm{~g}-\mathrm{p}$ ). It is important to note that the iNOS expression detected in the present model was primarily in macrophages, eosinophils and, to a lesser extent, in epithelial cells (fig. 1g-1). Deletion of the iNOS gene caused a moderate but significant reduction in eosinophil recruitment to airways upon OVA challenge (fig. 1a-f) and, as expected, no protein nitration was observed (fig. $1 \mathrm{~m}-\mathrm{p}$ ). These results suggest that iNOS and associated tissue damage (i.e. protein nitration) are necessary but insufficient for the full manifestation of eosinophilia upon OVA challenge in this animal model.

iNOS expression is required for oxidative DNA damage and concomitant PARP-1 activation upon allergen exposure

To determine whether a lack of iNOS-mediated tissue damage corresponded with a lowering in tissue oxidative DNA damage, IHC was performed on lung sections from OVAsensitised and challenged WT or $\mathrm{iNOS}^{-1-}$ mice with antibodies 

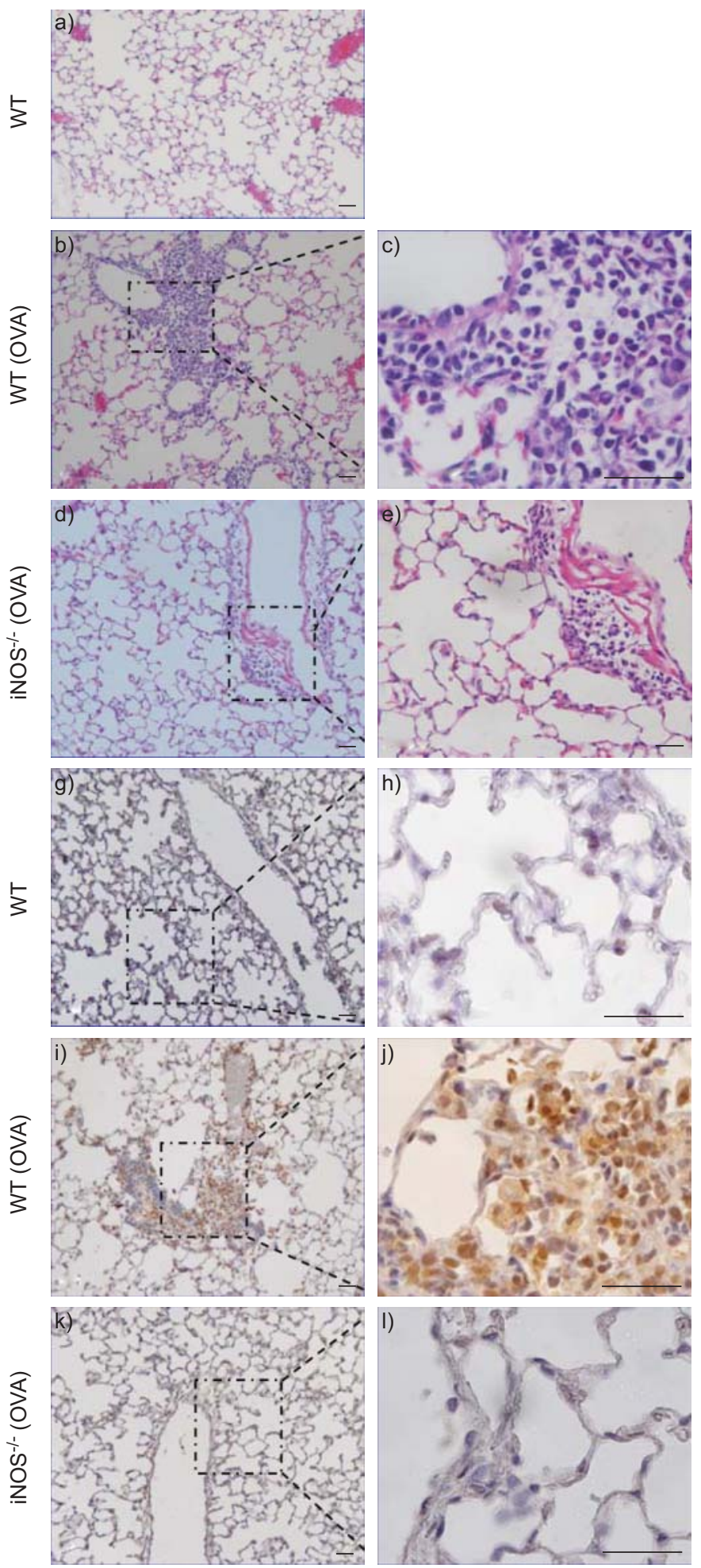
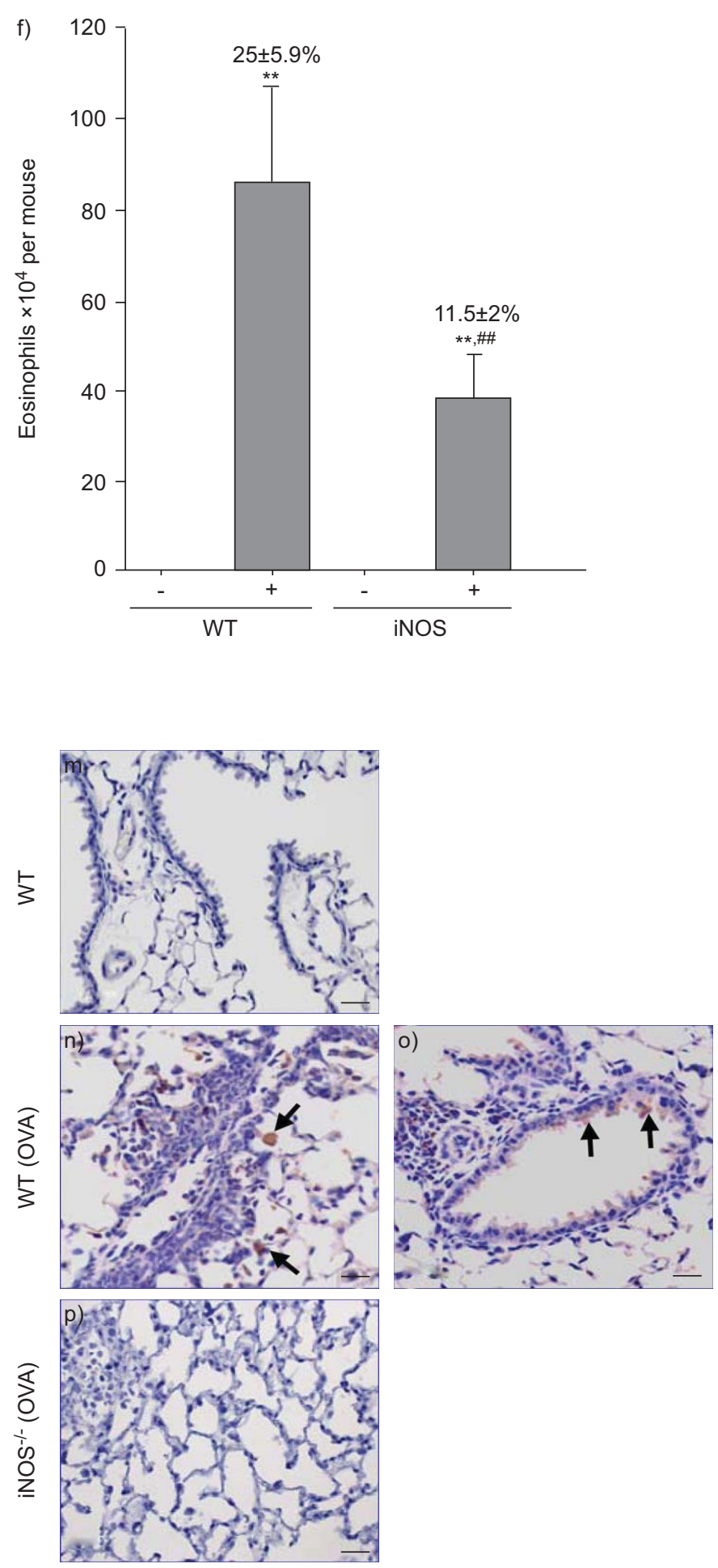

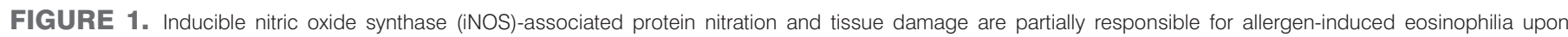

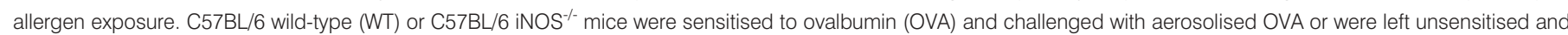

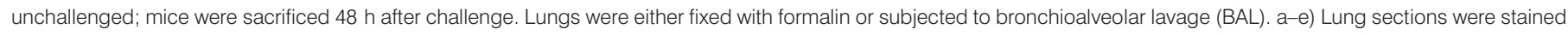

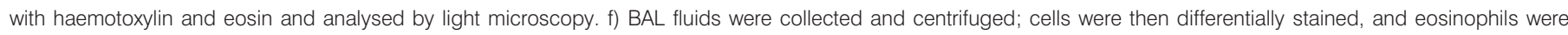

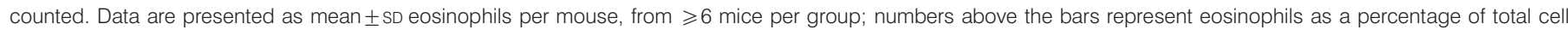

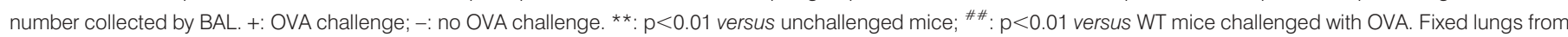

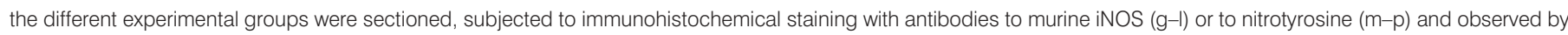

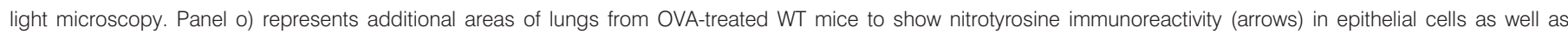
infiltrating inflammatory cells. Scale bars $=4 \mu \mathrm{m}$. 
to 8-oxo-7,8-dihydroguanine (8-oxodG) or to the poly(ADPribose) (PAR) moiety of PARP-1-modified proteins. As shown in figure $2 a-d$, in lung sections from OVA-challenged WT mice, 8-oxodG staining was prominent, but it was almost completely absent in lung sections from OVA-challenged iNOS $^{-/-}$mice. Figure $2 \mathrm{e}-\mathrm{g}$ shows that in OVA challenged mice, a marked PAR staining of WT lung tissue was observed which was nearly absent in iNOS $^{-/-}$mice. This establishes an association between the occurrence of oxidative DNA damage and PARP-1 activation upon OVA challenge, confirming previous reports [13]. More importantly, these results suggest that iNOS may be a central participant in mediating oxidative DNA damage, ultimately by activating PARP-1 in lung tissue upon allergen exposure.

\section{PARP-1 expression is required for iNOS expression and consequent protein nitration: a connection with NF- $\kappa B$ signal transduction}

The relationship between iNOS and PARP-1 was then further assessed by examining the effect of a PARP-1 gene deletion on iNOS expression and the consequence of such expression on tissue injury after allergen exposure. As shown in figure 3, a PARP-1 gene deletion, as previously reported [22], severely reduced eosinophil recruitment upon OVA challenge. It is important to note that the PARP-1 gene deletion exerted a higher impact on eosinophil recruitment compared with that exerted by deletion of the iNOS gene (compare data in figure $3 a$ with that in figure 1a-e). An IHC analysis shows that while iNOS was highly expressed in lungs of OVA-challenged WT mice, its expression was severely reduced in lungs of PARP-1 ${ }^{-/}$ mice, further supporting the notion that PARP-1 expression is important for efficient expression of iNOS (fig. 3b). Consequently, lung sections from OVA-challenged PARP-1 ${ }^{-/-}$ mice showed practically no staining for nitrotyrosine (fig. 3c) or 8-oxodG, indicating a complete absence of protein nitration and associated oxidative DNA damage as a result of a severe reduction in iNOS protein expression.

To determine whether PARP-1 regulates the expression of iNOS through the signal transduction pathways of NF- $\kappa \mathrm{B}$ in the experimental model, the activation of NF- $\mathrm{KB}$ in lung sections derived from OVA-challenged WT and PARP-1 ${ }^{-/-}$mice was examined using IHC. OVA challenge induced translocation of p65 NF- $\kappa B$ into the nuclei of a number of cell types (such as macrophages) in the lungs of WT mice (fig. $3 \mathrm{f}$ and $\mathrm{g}$ ). In contrast, p65 translocation was not apparent in the lungs of PARP-1 $1^{-/}$ mice (fig. 3h). Translocation of p $65 \mathrm{NF}-\kappa \mathrm{B}$ was not detected in cells of tissue sections derived from experimental groups that were not exposed to OVA (fig. 3f and data not shown).

OVA challenge induced phosphorylation of inhibitor of (I-) $\kappa \mathrm{B} \alpha$ in a number of lung cells in sections derived from WT mice, with many of them having macrophage features as assessed by IHC with antibodies to the phosphorylated form of $\mathrm{I}-\kappa \mathrm{B} \alpha$ (fig. 3i). It is important to note that NF- $\kappa \mathrm{B}$ activation has a transient nature, which justifies the lack of positive signal in all lung cells. In sharp contrast, OVA challenge of PARP-1/mice did not result in $\mathrm{I}-\kappa \mathrm{B} \alpha$ phosphorylation in lung tissues (fig. 3j). Taken together, these results suggest that the effect of a PARP-1 gene deletion on iNOS expression involves the signal transduction pathway of NF- $\mathrm{KB}$. Using primary macrophages and LPS as a stimulus, it was confirmed that a PARP-1 gene
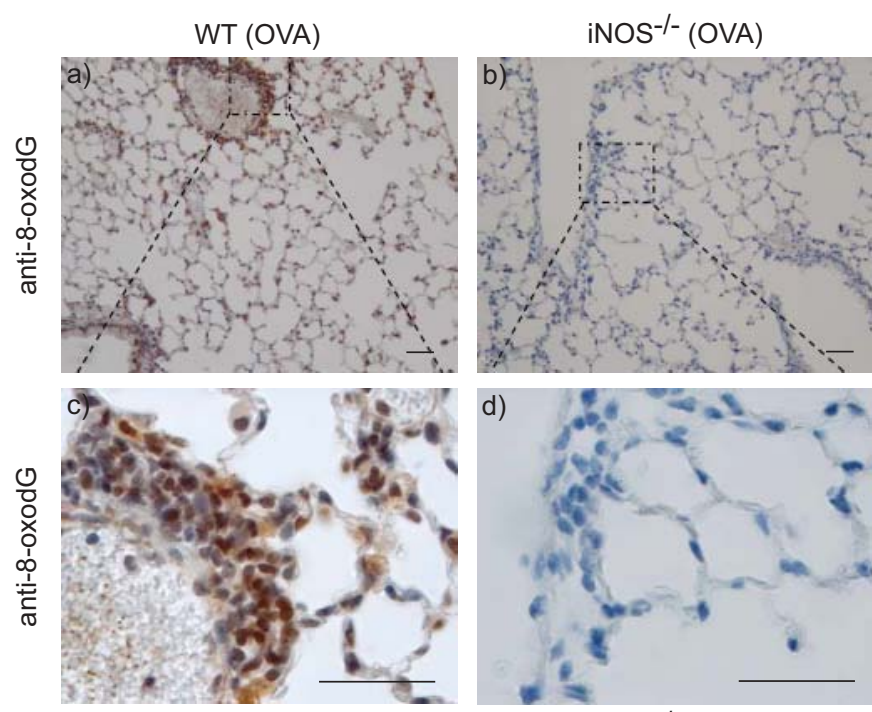
WT (OVA)

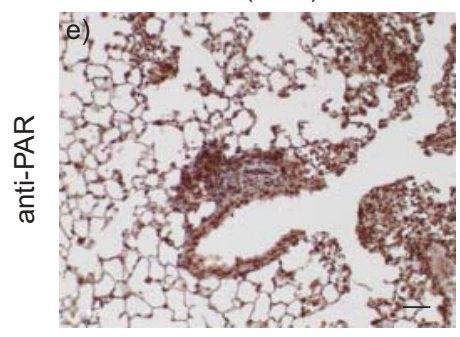

iNOS $^{-/-}($OVA $)$

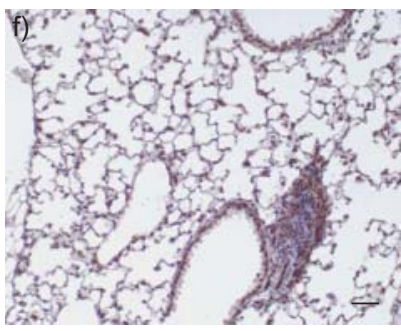

PARP-1/- (OVA)

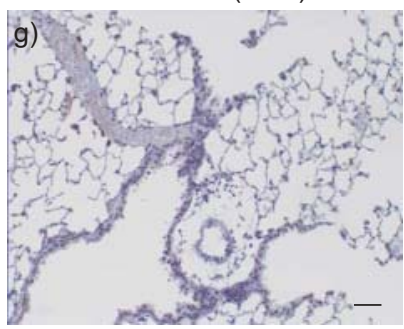

FIGURE 2. Inducible nitric oxide synthase (iNOS) expression is required for oxidative DNA damage and concomitant poly(ADP-ribose) polymerase-1 (PARP-1) activation upon allergen exposure. C57BL/6 wild-type (WT) and $\mathrm{NOS}^{-/-}$mice were subjected to ovalbumin (OVA) sensitisation and challenge or left unsensitised and unchallenged. Fixed lungs from the different experimental groups were sectioned, subjected to immunohistochemical staining with antibodies to 8-oxo-7,8-dihydroguanine (8-oxodG; $a-d)$ or to poly(ADP-ribose) moieties of PARP-1-modified proteins (PAR; e-g) and observed by light microscopy. g) Lung sections from OVAsensitised and challenged PARP- $1^{-1}$ mice were used as negative controls for poly(ADP-ribose) immunoreactivity. Scale bars $=4 \mu \mathrm{m}$.

deletion severely affected iNOS expression (fig. 3k) and that such an effect was associated with a defect in NF- $\kappa B$ nuclear translocation (fig. 31).

\section{By-products of iNOS enzymatic activity nitrate PARP-1 and inhibit its $N A D^{+}$-associated catalytic activity}

Protein nitration has been shown to result in the inactivation of a number of enzymes and transcription factors [26]. To determine whether PARP-1 can be modified by by-products of iNOS enzymatic activity, lung tissue extracts from control or 

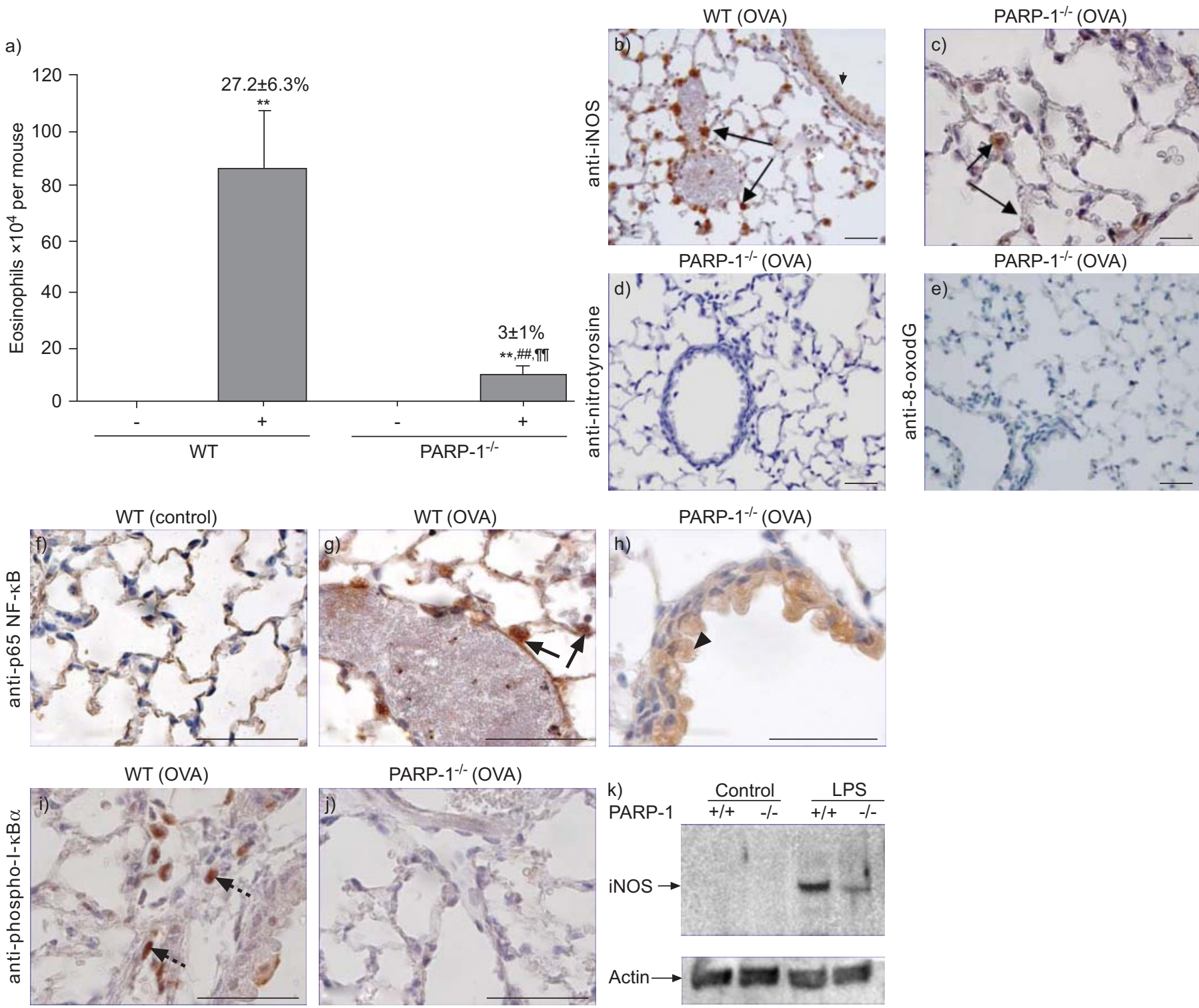

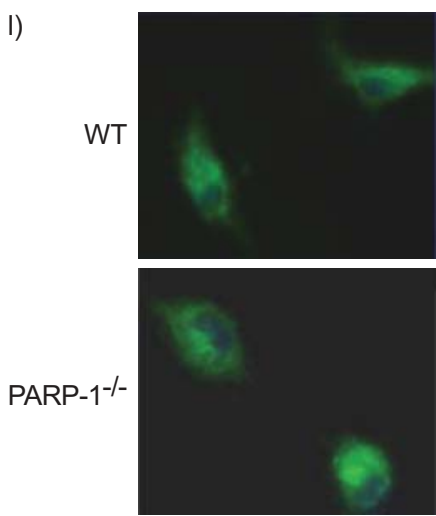

Control
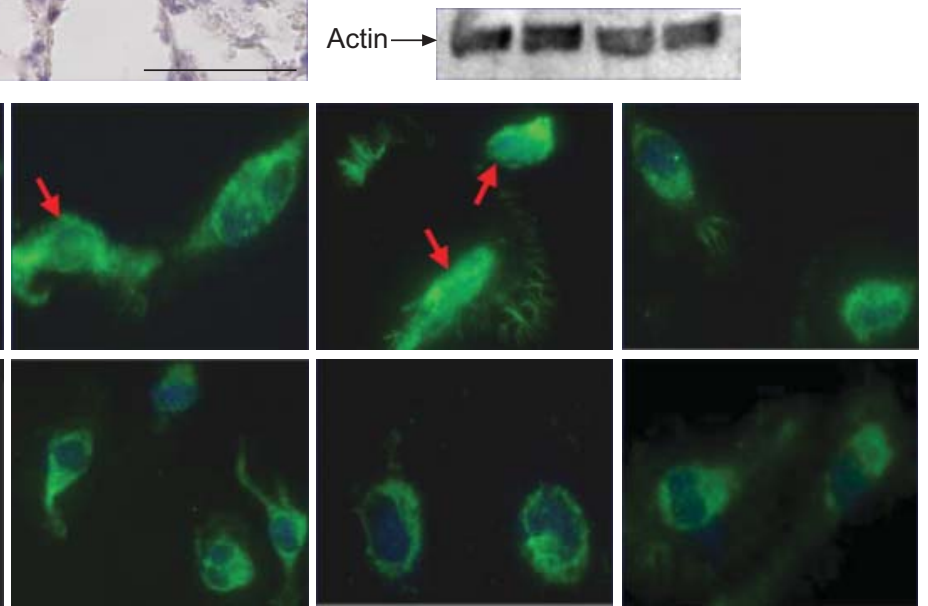

$30 \min$

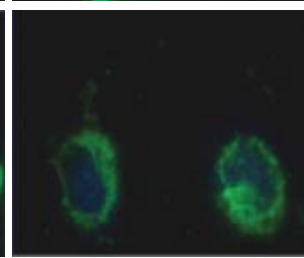

$1 \mathrm{~h}$

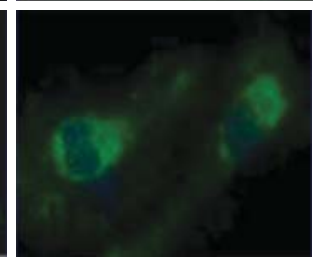

$4 \mathrm{~h}$

FIGURE 3. For legend see following page.

OVA-challenged mice were subjected to immunoprecipitation with antibodies to 3-nitrotyrosine, after which immunoprecipitates were subjected to immunoblot analysis with antibodies to mouse PARP-1. Figure 4a shows that OVA challenge did lead to nitration of PARP-1. To confirm that PARP-1 was nitrated by by-products of iNOS enzymatic activity, it was tested whether 
FIGURE 3. Poly(ADP-ribose) polymerase-1 (PARP-1) expression is required for inducible nitric oxide synthase (iNOS) expression and consequent protein nitration: a connection with nuclear factor (NF)-kB signal transduction. Wild-type (WT) or PARP-1\% mice were ovalbumin (OVA)-sensitised and challenged or left untreated. Mice were sacrificed $48 \mathrm{~h}$ after challenge and lungs were either subjected to bronchioalveolar lavage (BAL) or fixed with formalin for immunohistochemical (IHC) staining. a) BAL fluids were collected and centrifuged; cells were then differentially stained, and eosinophils were counted. Data are presented as mean \pm SD eosinophils per mouse, from $\geqslant 6$ mice per group; numbers above the bars represent eosinophils as a percentage of total cell number collected by BAL. ${ }^{*}: p<0.01$ versus unchallenged mice; $\# \#$ : $p<0.01$ versus

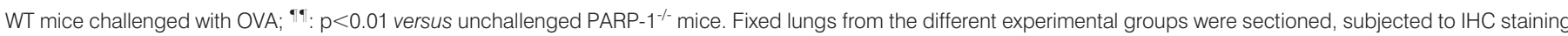
with antibodies to murine iNOS (b and c), to nitrotyrosine (d), or to 8-oxo-7,8-dihydroguanine (8-oxodG; e) and observed by light microscopy. IHC analysis of lung section from control mice is not shown. Fixed lungs from unchallenged WT mice (f), OVA-sensitised and challenged WT mice ( $\mathrm{g}$ and $\mathrm{i}$ ), or OVA-sensitised and challenged PARP-1 ${ }^{-1}$ mice ( $h$ and $j$ ) were sectioned, subjected to IHC staining with antibodies to murine p65 NF-kB (f-h) or phospho-inhibitor of $\kappa B \alpha$ (phospho-I-kB $\alpha$; i and j), and observed by light microscopy. The untreated controls, which exhibited no phospho-l-kB $\alpha$ immunoreactivity, are not shown. k) Macrophages isolated from peritoneal cavities of WT or PARP-1 ${ }^{-1}$ mice were treated with $1 \mathrm{mg} \cdot \mathrm{mL}^{-1}$ lipopolysaccharide (LPS) for $12 \mathrm{~h}$, after which protein extracts were prepared and subjected to immunoblot analysis with antibodies to murine iNOS or actin. Solid arrows: macrophages; dotted arrows: areas of immunoreactivity; arrowheads: endothelial cells. Scale bars $=4 \mu \mathrm{m}$. (I) WT or PARP-1/ macrophages were treated with $1 \mathrm{mg} \cdot \mathrm{mL}^{-1}$ lipopolysaccharide for the indicated times or left untreated (control) and were then subjected to immunofluorescence staining with antibodies to murine p65 NF-kB and 4'-6-diamidino-2-phenylindole. Arrows indicate NF-kB nuclear translocation.

direct exposure of purified recombinant PARP-1 to $\mathrm{ONOO}^{-}$in vitro would lead to PARP-1 nitration. Indeed, PARP-1 was an acceptor for nitration as assessed by immunoblot analysis with antibodies to 3-nitrotyrosine (fig. 4b), confirming the observation using the animal model. The consequence of such modification was then tested in vitro by assessing the ability of modified recombinant PARP-1 to poly(ADP-ribosyl)ate proteins derived from the liver of $\mathrm{PARP}-1^{-/-}$mice. Exposure of PARP-1 to $\mathrm{ONOO}^{-}$completely inactivated its enzymatic activity as evidenced by the absence of protein modification compared with samples that did not receive $\mathrm{ONOO}^{-}$(fig. 4c). Accordingly, this potential regulation of PARP-1 by byproducts of the enzymatic activity of iNOS may represent another facet of the regulatory relationship between PARP-1 and iNOS.

\section{iNOS expression following IL-5 production is dispensable for the establishment of eosinophilia but may be required for oxidative DNA damage and full inflammation-associated mucus production}

The present authors recently reported that IL-5 production may be dependent on PARP-1 expression [22]. Re-establishment of IL-5 through intranasal administration causes a reversal of eosinophilia in OVA-challenged $\mathrm{PARP}-1^{-/-}$mice (fig. 5a). This model enables investigation of whether the observed reversal in eosinophilia occurred concomitantly with iNOS expression. Reversal of eosinophilia by IL-5 replenishment in OVA-challenge PARP-1 ${ }^{-/-}$mice was not associated with a reversal of iNOS expression (fig. 5b) suggesting that iNOS expression may be dispensable after IL-5 production.

Oxidative DNA damage, such as the formation of 8-oxodG, is increasingly being associated with iNOS enzymatic activity in conditions other than allergen-induced inflammation [27-29]. The absence of a reversal of iNOS expression was accompanied with a complete absence of oxidative DNA damage (fig. 5e and f) and protein nitration (data not shown), which may suggest that iNOS expression may be an important participant in mediating oxidative DNA damage upon allergen exposure.

Hyperproduction of mucus by goblet cells is an important feature of asthma-associated airway inflammation that has been associated with the production of T-helper (Th)2 cytokines (for review, see [30]). Despite the fact that IL-5 replenishment reversed eosinophilia in OVA-challenged $\mathrm{PARP}^{-1^{-/}}$mice, it did not completely reverse mucus production (fig. $5 \mathrm{f}-\mathrm{i}$ ). Such a partial reversal may be related, in part, to iNOS deficiency. Taken together, these results suggest that iNOS may be dispensable for eosinophilia post-IL-5 production; however, it may participate in the manifestation of tissue damage during allergic airway inflammation.

\section{DISCUSSION}

While the function of iNOS in the process of airway inflammation has been addressed in a number of reports [31-34], its exact role remains elusive. This is due primarily to the different roles that its product $(\mathrm{NO})$ plays during inflammation. NO has been shown to function as a bronchodilator with anti-inflammatory properties, but it also has pro-inflammatory properties and can damage tissue through its ability to interact with superoxide to generate $\mathrm{ONOO}^{-}$(reviewed in $[35,36]$ ). Clinically, however, the level of exhaled NO has been reliably associated with severity of disease symptoms in asthmatics [3]. A few reports show a direct role for iNOS in the process of eosinophil recruitment in that inhibition of iNOS, either pharmacologically or by a gene knockout, reduces airway infiltration by inflammatory cells, especially eosinophils, in models of allergen-induced lung inflammation (reviewed in [25]). Furthermore, two very recent reports showed that polymorphisms in the iNOS gene may be important for asthma protection or susceptibility [11, 12]. However, the results of a recent clinical trial conducted by SINGH et al. [10] questioned the role of iNOS during asthma and the viability of iNOS as a therapeutic target to treat the symptoms of the disease.

The current study examined the role of iNOS in the pathogenesis of allergen-induced airway inflammation by analysing the relationship of this enzyme with PARP-1, a DNA damageresponsive enzyme and another important participant in inflammation. The results of the present study propose a reciprocal relationship between the two proteins that involves a requirement of PARP-1 for iNOS expression, activation of PARP-1 by oxidative DNA damage caused by iNOS byproducts, and a modulation of PARP-1 enzymatic activity by nitration. Additionally, the data suggest that iNOS may be dispensable after IL-5 production (see scheme in fig. 6). These results may shed a preliminary light on the recently reported ineffectiveness of a specific iNOS inhibitor in preventing allergen-induced asthma in humans [10] given the potential production of large quantities of Th2 cytokines such as IL-5 as a result of repeated exposure to allergens. Accordingly, the current 
a) OVA

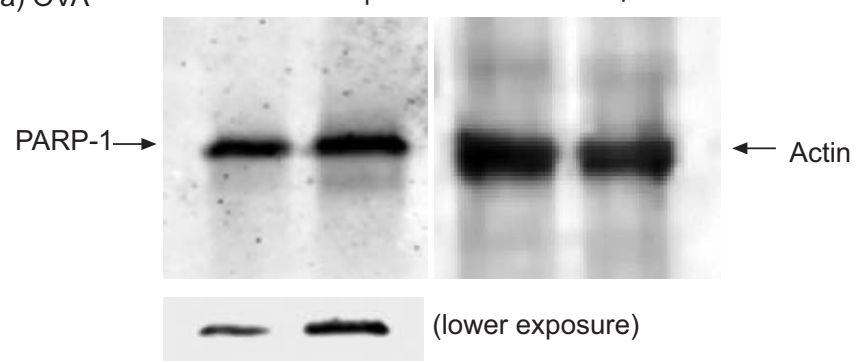

b) $\mathrm{ONOO}^{-}$

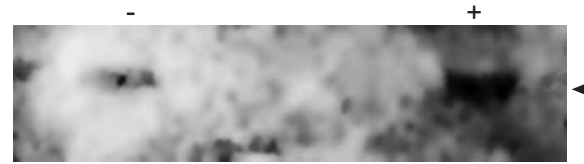

c) PARP-1 $\mathrm{ONOO}^{-}$

PARP-1

(nitrated)
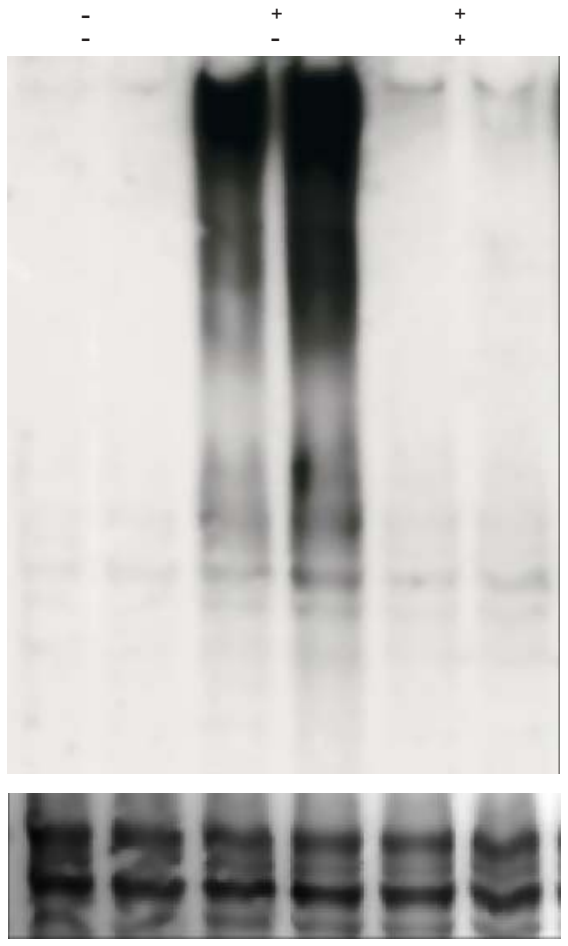

FIGURE 4. By-products of inducible nitric oxide synthase enzymatic activity nitrate poly(ADP-ribose) polymerase-1 (PARP-1) and inhibit its enzymatic activity (i.e. poly(ADP-ribosyl)ation). a) Lung extracts (150 $\mu \mathrm{g}$ ) from untreated or wild-type mice subjected to ovalbumin (OVA)-sensitisation and challenged and sacrificed $24 \mathrm{~h}$ after challenge were subjected to immunoprecipitation with antibodies to nitrotyrosine, after which the precipitates were subjected to immunoblot analysis with antibodies to PARP-1. The lower panel represents a lower exposure of the blot to show the clear difference between the two samples. A $10 \%$ sample input was subjected to immunoblot with antibodies to actin to demonstrate equal loading (right panel). b) Recombinant human PARP-1 was incubated in a nitration reaction mixture containing $10 \mu \mathrm{M}$ peroxynitrite (ONOO) for $10 \mathrm{~min}$, after which the samples were subjected to immunoblot analysis with antibodies to nitrotyrosine. c) Recombinant PARP-1, treated with $10 \mu \mathrm{M}$ ONOO or left untreated as in (b), in duplicates, was incubated with equal amounts of liver protein extracts derived from PARP-1\% mice (see Ponceau stain, d) and subjected to an in vitro poly(ADP-ribosyl)ation reaction with nicotinamide adenine dinucleotide and activated (sonicated) DNA for 5 min. The reactions were terminated with sample buffer and subjected to immunoblot analysis with antibodies to poly(ADP-ribose).

study provides new information about the intricate relationship between iNOS and allergen-induced inflammation and also highlights the need to think of new strategies for using iNOS as a therapeutic target for the treatment of asthma symptoms.

Induction of iNOS expression and the subsequent production of $\mathrm{NO}$ in the lungs after allergen exposure has been of particular interest in numerous asthma-related studies [3, 37]. It is important to note that $\mathrm{NO}$ can also be produced by the other two isoforms of NOS (eNOS and nNOS) [37]. The current study shows that, in an animal model, iNOS is expressed primarily in macrophages, eosinophils and epithelial cells as well as in smooth muscle cells (data not shown). Additional cellular sources of $\mathrm{NO}$ in the lung may include endothelial cells of pulmonary arteries and veins, inhibitory nonadrenergic noncholinergic neurones, mast cells, mesothelial cells, fibroblasts, neutrophils and lymphocytes [35, 37]. It is presumed that NO produced by eNOS and nNOS may play a beneficial role, while NO produced by iNOS may be detrimental [37]. This difference may be associated with the level of NO produced rather than its cellular or molecular source. Despite the clear induction of iNOS expression in the airways after allergen exposure, its role in the pathogenesis of asthma in animal models of the disease has been challenged by several studies (for a detailed review, see [25]). The current study provides evidence for a close relationship between PARP-1 and iNOS during airway inflammation. Indeed, maximal induction of iNOS in response to allergen exposure in vivo or to LPS treatment in vitro was highly dependent on PARP-1 expression. Re-establishment of PARP-1 expression using adenovirus-mediated gene transfer reversed iNOS expression in a cell culture system (data not shown), confirming this requirement. NF- $\mathrm{B}$ signal transduction appears to be necessary for iNOS expression as it is affected by a PARP-1 gene deletion. Recently, YU et al. [38] showed in an elegant study that PARP-1 binds to the promoter of the iNOS gene and as a feedback regulation mechanism, PARP-1 is S-nitrosylated, thus removing its ability to bind the iNOS gene promoter, leading to a downregulation of iNOS expression. However, iNOS gene downregulation may also involve an important feedback mechanism with $\mathrm{I}-\kappa \mathrm{B}$ proteins that specifically bind $\mathrm{NF}-\kappa \mathrm{B}$ and prevent the binding of the transcription factor to the iNOS gene promoter [39]. Furthermore, the present results show that PARP-1 modification by nitration severely inhibits its enzymatic activity, unravelling a new facet of regulation by iNOS by-products on PARP-1. Evidently, such inhibition may exert numerous outcomes during inflammation. It may not only regulate expression of a number of cytokines and factors that are dependent on PARP-1, but may also modulate the response of PARP-1 to DNA-damaging agents such as 
PARP $-1^{-/-}$+saline (OVA)

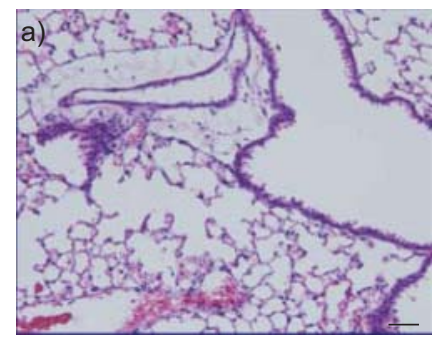

PARP-1/- + IL-5 (OVA)

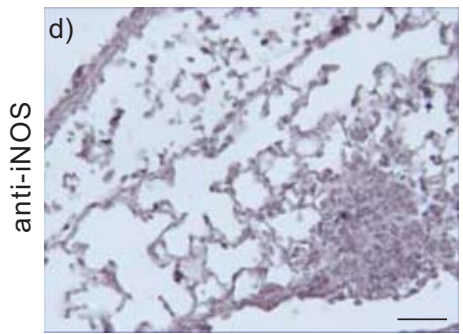

WT + saline (OVA)
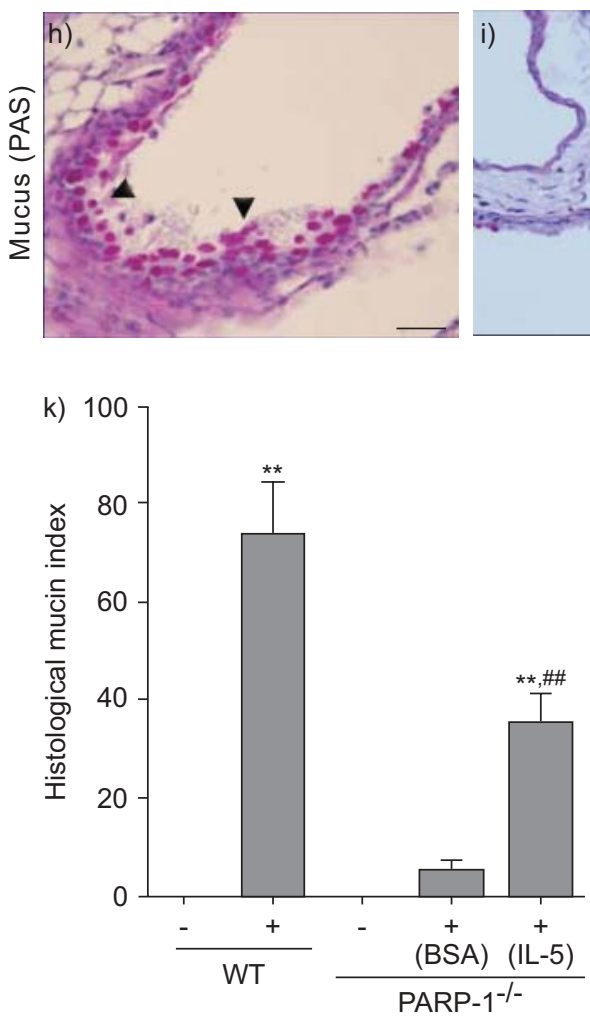

PARP-1/- +IL-5 (OVA)

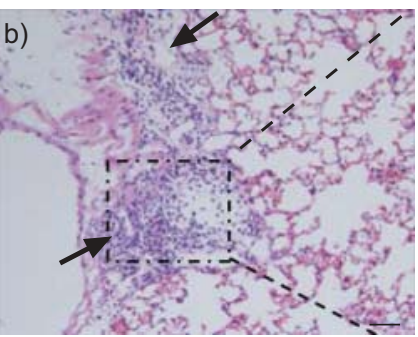

WT (OVA)

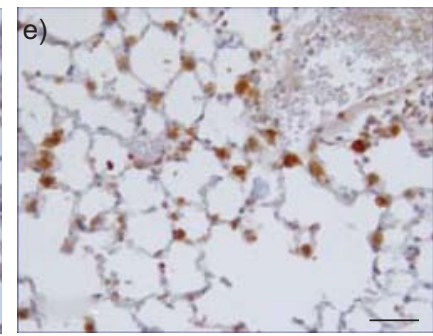

PARP $-1^{-/-}(\mathrm{OVA})$

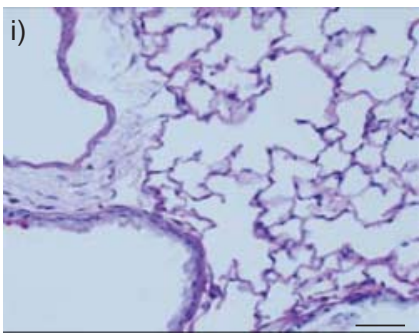

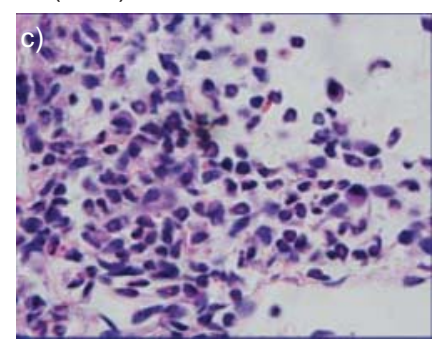

PARP $-1^{-/-}+$IL-5 (OVA)
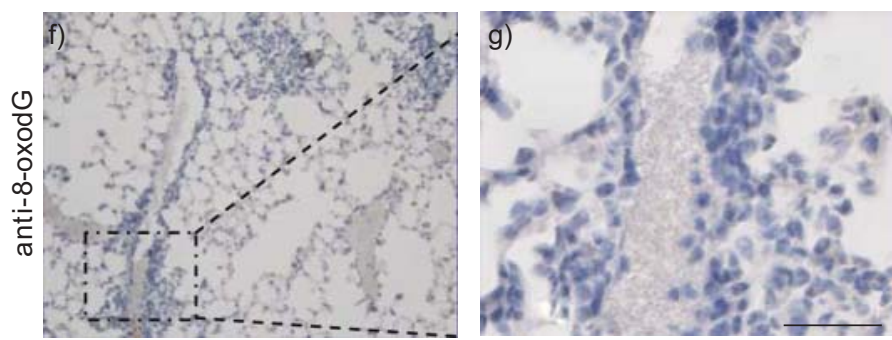

PARP-1/- + IL-5 (OVA)

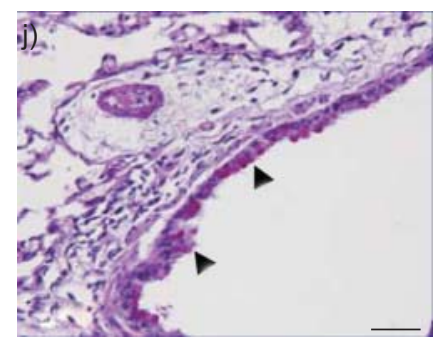

FIGURE 5. Expression of inducible nitric oxide synthase (iNOS) is dispensable after interleukin (IL)-5 production for the establishment of eosinophilia and associated oxidative DNA damage but is required for full manifestation of inflammation-associated mucus production. Poly(ADP-ribose) polymerase-1 (PARP-1) - $^{-1}$ mice were sensitised to and challenged with ovalbumin (OVA). After $24 \mathrm{~h}$, mice were subjected to intranasal administration of recombinant mouse IL-5 $(0.5 \mu \mathrm{g})$, saline, or bovine serum albumin (BSA). Mice were sacrificed $48 \mathrm{~h}$ after the OVA challenge. $\mathrm{a}$ and $\mathrm{b}$ ) Fixed lungs from the different experimental groups were sectioned and subjected to haematoxylin and eosin staining; arrows indicate eosinophil infiltration. c) Enlargement of (b). $d$ and e) Sections from OVA-challenged PARP $-1^{-\%}$ mice that were subjected to intranasal administration of recombinant IL-5 (d) were subjected to immunohistochemical $(\mathrm{IHC})$ staining with antibodies to murine iNOS sections from OVA-challenged wild-type (WT) mice (e) were used as positive controls for iNOS expression. f) Lung sections from OVA-challenged PARP- $1^{-1-}$ mice that received an intranasal administration of recombinant IL-5 were subjected to IHC staining with antibodies to 8-oxo-7,8-dihydroguanine (8-oxodG). g) Enlargement of (f). $h-j)$ Lung sections from the latter group were subjected to periodic acid-Schiff (PAS) staining; sections from OVA-challenged WT mice were used as positive controls for mucus production. Arrowheads indicate sites of inflammatory cell infiltration or PAS-positive goblet cells. k) The extent of mucus production (histological mucin index) was assessed using Image-Pro Plus software. ${ }^{* *}: p<0.01$ versus unchallenged mice; ${ }^{\# \#}: p<0.01$ versus WT mice challenged with OVA. Scale bars $=4 \mu \mathrm{m}$.

oxidative stress, including that mediated by $\mathrm{ONOO}^{-}$. It is tempting to speculate that such PARP-1 inhibition by nitration may be considered as a feedback mechanism by which tissue and cells block continuity and persistence of inflammation.

It is noteworthy that iNOS gene deletion did not fully prevent PARP-1 activation, although it almost completely blocked the generation of 8-oxodG-related oxidative DNA damage. This result suggests that iNOS expression and the concomitant oxidative DNA damage are only partially effects of the observed PARP-1 activation after OVA challenge. The source of the additional DNA damage resulting from the remaining
PARP-1 activation is currently unknown but could very well be associated with the activities of additional oxidative stressgenerating enzymes such as myeloperoxidases or nicotinamide adenine dinucleotide phosphate-oxidase.

Recently, IJIMA et al. [32] reported an interesting connection between IL-5 and iNOS, as systemic administration of anti-IL-5 antibodies after OVA exposure was found to markedly inhibit iNOS expression and the consequent nitration of proteins in an animal model similar to that used in the current study. These findings suggest that IL-5 may induce iNOS expression. But it is not clear whether such a reduction in iNOS expression is a 


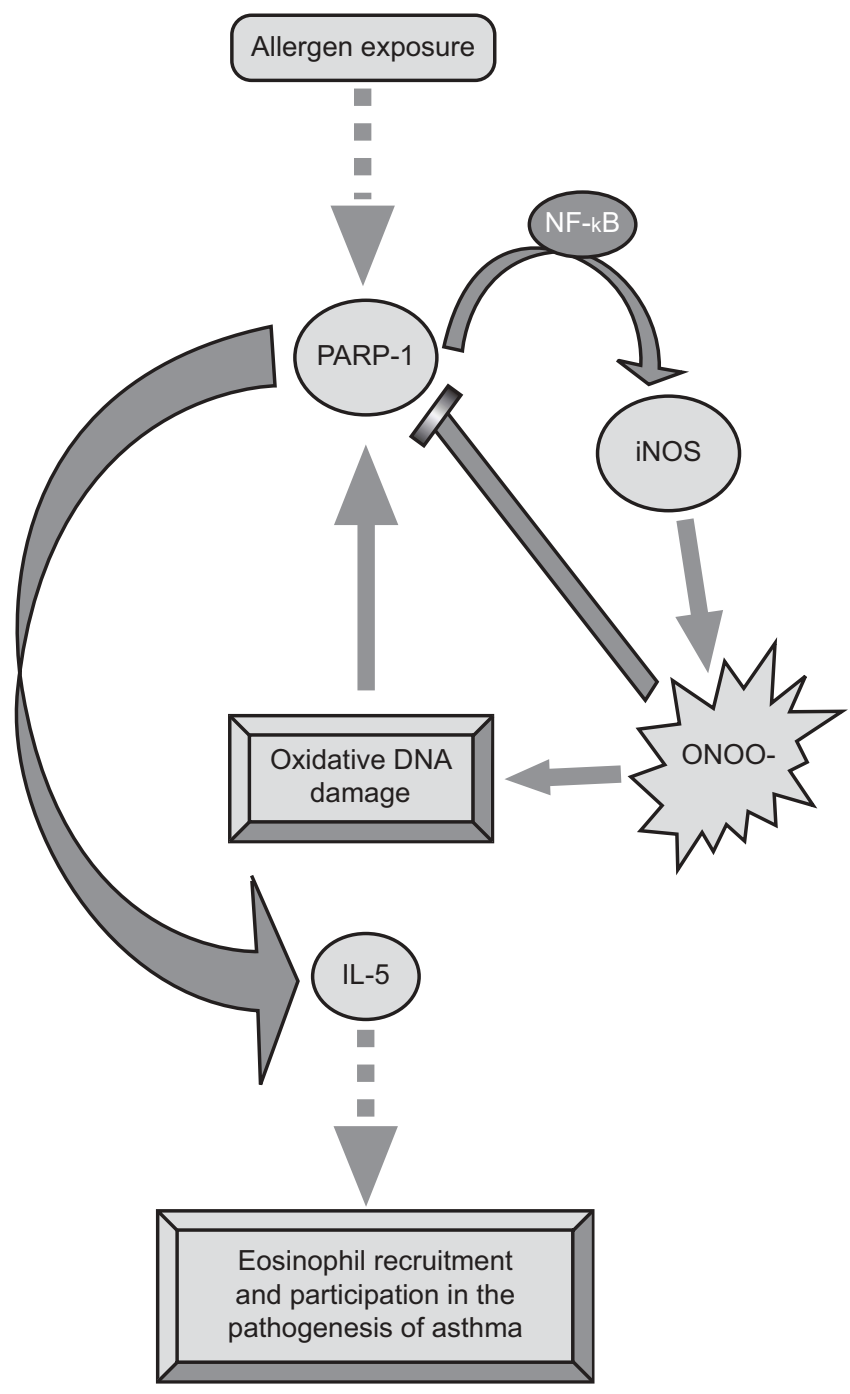

FIGURE 6. Model for the potential reciprocal regulation of inducible nitric oxide synthase (iNOS) and poly(ADP-ribose) polymerase-1 (PARP-1) during allergeninduced airway inflammation. Upon allergen exposure that involves a number of intricate processes, PARP-1 participates in the process of iNOS expression potentially through nuclear factor (NF)-kB-mediated signal transduction and interleukin (IL)-5 production through a yet-unknown mechanism. IL-5, in addition to several important cytokines, promotes the recruitment of inflammatory cells such as eosinophils to the lung. iNOS produces high levels of nitric oxide, which can be converted into peroxynitrite ( $\left.\mathrm{ONOO}^{-}\right)$after its interaction with superoxide. ONOOcauses oxidative tissue damage as manifested by protein nitration and induces DNA strand breaks which are potent activators of the nicotinamide adenine dinucleotide-utilising enzymatic activity of PARP-1. Nitration of PARP-1 renders the enzyme inactive, representing a potential regulatory mechanism by which iNOS modulates PARP-1 enzymatic activity. Inhibition of PARP-1 may represent an attempt by the cells to control the inflammatory response.

consequence of direct cross-talk between IL-5 and iNOS or an indirect effect, either through a reduction in eosinophil recruitment or by reducing the overall production of reactive oxygen species. The present results show that administration of recombinant IL-5 was sufficient to cause eosinophil recruitment in OVA-challenged PARP-1/- mice but was insufficient to drive iNOS expression. This suggests that iNOS expression is not directly dependent on IL-5, but that iNOS participates in eosinophilia by upregulating IL-5 in the current model (data not shown). Furthermore, the requirement of iNOS for the establishment of eosinophilia may be bypassed by IL-5. However, this does not preclude a role for iNOS in the damage that may be caused by iNOS expression and its by-products after eosinophil recruitment. Indeed, the current results show that iNOS may be crucial in order for oxidative tissue damage and mucus production to take place. Accordingly, the current results provide potential additional association between iNOS and the process of allergic airway inflammation.

Taken together, the present results support the existence of an important reciprocal relationship between poly(ADP-ribose) polymerase- 1 and inducible nitric oxide synthase with a special connection to oxidative DNA damage and interleukin-5 in the process of eosinophilia during allergen-induced lung inflammation. Additional studies of these relationships may contribute to the clarification of the intricate role(s) of inducible nitric oxide synthase in asthma pathogenesis and may help in the design of new therapeutic strategies for the treatment of the condition.

\section{ACKNOWLEDGEMENTS}

The current authors would like to acknowledge the assistance of D. Siddiqui and would like to thank L. Harrison-Bernard for allowing them to use Image-Pro Plus.

\section{REFERENCES}

1 Seimon TA, Obstfeld A, Moore KJ, Golenbock DT, Tabas I. Combinatorial pattern recognition receptor signaling alters the balance of life and death in macrophages. Proc Natl Acad Sci USA 2006; 103: 19794-19799.

2 DeNicola LR, Kissoon N, Duckworth LJ, Blake KV, Murphy SP, Silkoff PE. Exhaled nitric oxide as an indicator of severity of asthmatic inflammation. Pediatr Emerg Care 2000; 16: 290-295.

3 Ricciardolo FL, Nijkamp FP, Folkerts G. Nitric oxide synthase (NOS) as therapeutic target for asthma and chronic obstructive pulmonary disease. Curr Drug Targets 2006; 7: 721-735.

4 MacNee W. Oxidative stress and lung inflammation in airways disease. Eur J Pharmacol 2001; 429: 195-207.

5 Kirkham P, Rahman I. Oxidative stress in asthma and COPD: antioxidants as a therapeutic strategy. Pharmacol Ther 2006; 111: 476-494.

6 Hogg JC. The pathology of asthma. APMIS 1997; 105: 735-745.

7 Hansel TT, Kharitonov SA, Donnelly LE, et al. A selective inhibitor of inducible nitric oxide synthase inhibits exhaled breath nitric oxide in healthy volunteers and asthmatics. FASEB J 2003; 17: 1298-1300.

8 Mulrennan SA, Redington AE. Nitric oxide synthase inhibition: therapeutic potential in asthma. Treat Respir Med 2004; 3: 79-88.

9 Brindicci C, Ito K, Barnes PJ, Kharitonov SA. Effect of an inducible nitric oxide synthase inhibitor on differential flow-exhaled nitric oxide in asthmatic patients and healthy volunteers. Chest 2007; 132: 581-588.

10 Singh D, Richards D, Knowles RG, et al. Selective inducible nitric oxide synthase inhibition has no effect on allergen challenge in asthma. Am J Respir Crit Care Med 2007; 176: 988-993. 
11 Holla LI, Stejskalova A, Znojil V, Vasku A. Analysis of the inducible nitric oxide synthase gene polymorphisms in Czech patients with atopic diseases. Clin Exp Allergy 2006; 36: 1592-1601.

12 Batra J, Singh TP, Mabalirajan U, Sinha A, Prasad R, Ghosh B. Association of inducible nitric oxide synthase (iNOS) with asthma severity, total serum IgE and blood eosinophil levels. Thorax 2006; 62: 16-22.

13 Boulares AH, Zoltoski AJ, Sherif ZA, Jolly P, Massaro D, Smulson ME. Gene knockout or pharmacological inhibition of poly(ADP-ribose) polymerase-1 prevents lung inflammation in a murine model of asthma. Am J Respir Cell Mol Biol 2003; 28: 322-329.

14 Suzuki Y, Masini E, Mazzocca C, et al. Inhibition of poly(ADP-ribose) polymerase prevents allergen-induced asthma-like reaction in sensitized Guinea pigs. J Pharmacol Exp Ther 2004; 311: 1241-1248.

15 Boulares H, Zoltoski AJ, Kandan S, Akbulut T, Yakovlev A, Oumouna M. Correlation between decreased sensitivity of the Daudi lymphoma cells to VP-16-induced apoptosis and deficiency in DNAS1L3 expression. Biochem Biophys Res Commun 2006; I341: 653-662.

16 Naura AS, Hans CP, Zerfaoui M, et al. Post-allergen challenge inhibition of poly(ADP-ribose) polymerase harbors therapeutic potential for treatment of allergic airway inflammation. Clin Exp Allergy 2008; 38: 839-846.

17 Cuzzocrea S. Shock, inflammation and PARP. Pharmacol Res 2005; 52: 72-82.

18 Pieper AA, Verma A, Zhang J, Snyder SH. Poly (ADPribose) polymerase, nitric oxide and cell death. Trends Pharmacol Sci 1999; 20: 171-181.

19 Tentori L, Portarena I, Graziani G. Potential clinical applications of poly(ADP-ribose) polymerase (PARP) inhibitors. Pharmacol Res 2002; 45: 73-85.

20 Hassa PO, Hottiger MO. The functional role of poly(ADPribose)polymerase 1 as novel coactivator of NF- $\mathrm{KB}$ in inflammatory disorders. Cell Mol Life Sci 2002; 59: 1534-1553.

21 Hassa PO, Hottiger MO. A role of poly (ADP-ribose) polymerase in NF- $\kappa \mathrm{B}$ transcriptional activation. Biol Chem 1999; 380: 953-959.

22 Oumouna M, Datta R, Oumouna-Benachour $\mathrm{K}$, et al. PARP-1 inhibition prevents eosinophil recruitment by modulating Th2 cytokines in a murine model of allergic airway inflammation: a potential specific effect on IL-5. J Immunol 2006; 177: 6489-6496.

23 Boulares AH, Yakovlev AG, Ivanova $\mathrm{V}$, et al. Role of poly(ADP-ribose) polymerase (PARP) cleavage in apoptosis. Caspase 3-resistant PARP mutant increases rates of apoptosis in transfected cells. J Biol Chem 1999; 274: 22932-22940.

24 Boulares HA, Giardina C, Navarro CL, Khairallah EA, Cohen SD. Modulation of serum growth factor signal transduction in Hepa 1-6 cells by acetaminophen: an inhibition of c-myc expression, NF- $\mathrm{KB}$ activation, and Raf-1 kinase activity. Toxicol Sci 1999; 48: 264-274.

25 Mathrani VC, Kenyon NJ, Zeki A, Last JA. Mouse models of asthma: can they give us mechanistic insights into the role of nitric oxide? Curr Med Chem 2007; 14: 2204-2213.

26 Bruckdorfer R. The basics about nitric oxide. Mol Aspects Med 2005; 26: 3-31.

27 Murata M, Kawanishi S. Oxidative DNA damage induced by nitrotyrosine, a biomarker of inflammation. Biochem Biophys Res Commun 2004; 316: 123-128.

28 Pinlaor S, Hiraku Y, Ma N, et al. Mechanism of NOmediated oxidative and nitrative DNA damage in hamsters infected with Opisthorchis viverrini: a model of inflammation-mediated carcinogenesis. Nitric Oxide 2004; 11: 175-183.

29 Pinlaor S, Hiraku Y, Yongvanit P, et al. iNOS-dependent DNA damage via NF- $\kappa$ B expression in hamsters infected with Opisthorchis viverrini and its suppression by the antihelminthic drug praziquantel. Int J Cancer 2006; 119: 1067-1072.

30 Rogers DF. Physiology of airway mucus secretion and pathophysiology of hypersecretion. Respir Care 2007; 52: 1134-1146.

31 Xiong Y, Karupiah G, Hogan SP, Foster PS, Ramsay AJ. Inhibition of allergic airway inflammation in mice lacking nitric oxide synthase 2. J Immunol 1999; 162: 445-452.

32 Iijima H, Duguet A, Eum SY, Hamid Q, Eidelman DH. Nitric oxide and protein nitration are eosinophil dependent in allergen-challenged mice. Am J Respir Crit Care Med 2001; 163: 1233-1240.

33 Eynott PR, Paavolainen N, Groneberg DA, et al. Role of nitric oxide in chronic allergen-induced airway cell proliferation and inflammation. J Pharmacol Exp Ther 2003; 304: 22-29.

34 Bhandari V, Choo-Wing R, Chapoval SP, et al. Essential role of nitric oxide in VEGF-induced, asthma-like angiogenic, inflammatory, mucus, and physiologic responses in the lung. Proc Natl Acad Sci USA 2006; 103: 11021-11026.

35 Dweik RA. Nitric oxide, hypoxia, and superoxide: the good, the bad, and the ugly! Thorax 2005; 60: 265-267.

36 Redington AE. Modulation of nitric oxide pathways: therapeutic potential in asthma and chronic obstructive pulmonary disease. Eur J Pharmacol 2006; 533: 263-276.

37 Ricciardolo FL, Sterk PJ, Gaston B, Folkerts G. Nitric oxide in health and disease of the respiratory system. Physiol Rev 2004; 84: 731-765.

38 Yu Z, Kuncewicz T, Dubinsky WP, Kone BC. Nitric oxidedependent negative feedback of PARP-1 trans-activation of the inducible nitric-oxide synthase gene. J Biol Chem 2006; 281: 9101-9109.

39 Chen LF, Greene WC. Regulation of distinct biological activities of the NF- $\kappa \mathrm{B}$ transcription factor complex by acetylation. J Mol Med 2003; 81: 549-557. 